\title{
On the Alternative Approach to Active Control
}

\author{
Vladimir V. Arabadzhi \\ Division of Geophysical Research, Institute of Applied Physics (RAS), Nizhny Novgorod, Russia \\ Email: v.v.arabadzhi@appl.sci-nnov.ru
}

How to cite this paper: Arabadzhi, V.V. (2018) On the Alternative Approach to Active Control. Journal of Applied Mathematics and Physics, 6, 2303-2312. https://doi.org/10.4236/jamp.2018.611191

Received: September 20, 2018 Accepted: November 19, 2018 Published: November 22, 2018

\begin{abstract}
Paper is devoted to problem of acoustical visibility reduction and gives brief description of alternative approach to active control. This approach allows satisfy jointly the four conditions: a) effective suppression of acoustical radiation and scattering caused by elastic body immersed in compressible medium (water); b) cloacking at any important temporal frequencies of observant sound waves; c) cloacking at any important spatial frequencies or at any observation directions; (d) thickness of the masking shell is much smaller than the length of suppressed waves. Technological development gives more and more fast and miniature control elements and fast, accurate calculations. On the other hand, the lengths of waves to be damped are constant due to the constant conditions of their far propagation. The approach suggested uses operations of high space-time resolution for long waves controlling. Because the joint fulfillment of the conditions of acoustic support absence and the thinness of the shell and also the causality of control lead to the need to operate at frequencies of much higher than frequencies of waves to be damped. It is assumed that the incident waves are represented by a discrete set of plane waves of finite duration. Also it is assumed only that the characteristic spatial scale of the distribution of normal velocities on the surface of the protected body is limited from below. The boundary value problem with initial conditions is considered.
\end{abstract}

\section{Keywords}

Boundary and Initial Conditions, Flat Impact, Flat Imprint, Imprint Relaxation, Depth of Imprint, Transparent Supportless Unidirectional Sources of Acoustical Wavelets, Leading Edge of Incident Wave, Leading Pair of Microphones

\section{Introduction}

Modern results [1] in the field of cloacking correlate with the famous article [2]. In this paper, an analytical solution is obtained in the case when the incident 
wave is flat and monochromatic, and the body has a spherical shape. The solution of this problem is tangentially homogeneous radial distribution of medium parameters in the outer spherical layer (that is the time-constant distribution of the passive parameters of the medium in the masking shell). Thus, the distribution of parameters required for the masking inside the protective layer depends on the frequency of the incident wave and does not depend on the direction of arrival of the incident wave. The need to adjust the spatial structure of the masking shell to a nonplanar form and the given direction of the incident wave (or to the non-spherical shape of the body) even in the monochromatic case, makes the practical application of the solution extremely complicated. Moreover the dynamic range of mechanical vibrations of the elements of the masking shell can be significantly exceeded, since all the power of the incident wave through the cross section of the protected body must tangentially pass inside the shell, the thickness of which we seek to reduce (see abstract, (d)). In addition, the above-described shell is not designed to suppress the radiation of the protected body. We will consider below the possibilities of radiation and scattering suppressing for all directions of observation and for all directions of the incident waves in the ranges $\omega_{\min } \leq \omega \leq \omega_{\max }$ and

$2 \pi c / \omega_{\max }=\lambda_{\min } \leq \lambda \leq \lambda_{\max }=2 \pi c / \omega_{\min }$ of frequencies and wavelengths (where $\omega_{\min }, \omega_{\max }, \lambda_{\min }, \lambda_{\max }$ sound damping range boundaries, $c$-sound speed in outside compressible medium) under the broadband $\omega_{\min }<<\omega_{\max }$, $\Xi / \lambda_{\min }>>1$, and $\Xi / \lambda_{\max }<<1$, where $\Xi$-characteristic linear dimension of body protected with convex smooth surface $S_{B}$ ) and small thickness $2 h$ of the active shell (coating between inner and outer surfaces $S_{B}$ and $S$ ) of the protected body in comparison with the lengths of quenched waves, i.e. $2 h<<\lambda_{\min }$. And the forced condition of inflexibility of surface $S_{B}$ for spatial scales $\ell_{\min }>>\lambda_{\min } / 2$ on any frequency $\omega$.

\section{Radiation Suppression}

In the most general formulation, we need to create on the active shell surface $S$ a predetermined distribution $U_{\mathbf{*}}(\mathbf{r}, t) \quad(\mathbf{r} \in S)$ of the normal displacements of particles, despite the action of unknown sources of vibrations inside the surface $S_{B}$. In particular, to suppress radiation, we need to create $U_{\bullet}(\mathbf{r}, t)=0$, i.e. no incident waves.

\subsection{Shock Molding of Desired Boundary Form}

Let us consider briefly the memory of a compressible or elastic linear medium about impact action, or, in other words, the formulation of a problem maximally different from a monochromatic case [2]. For a longitudinal impact to the free end $x=0$ of a semi-infinite elastic rod $(0 \leq x<\infty$, one dimensional problem, Figure 1(a)), the depth $Y=(\rho c)^{-1} \int_{0}^{\tau_{F}} F_{\#}(t) d t$ (where $\rho$-mass density of elastic rod, $c$-speed of longitudinal sound in the rod) of imprint of the rod end remains in time infinitely (imprint life-time $\tau_{\#}=\infty$ ) after the switching off the 

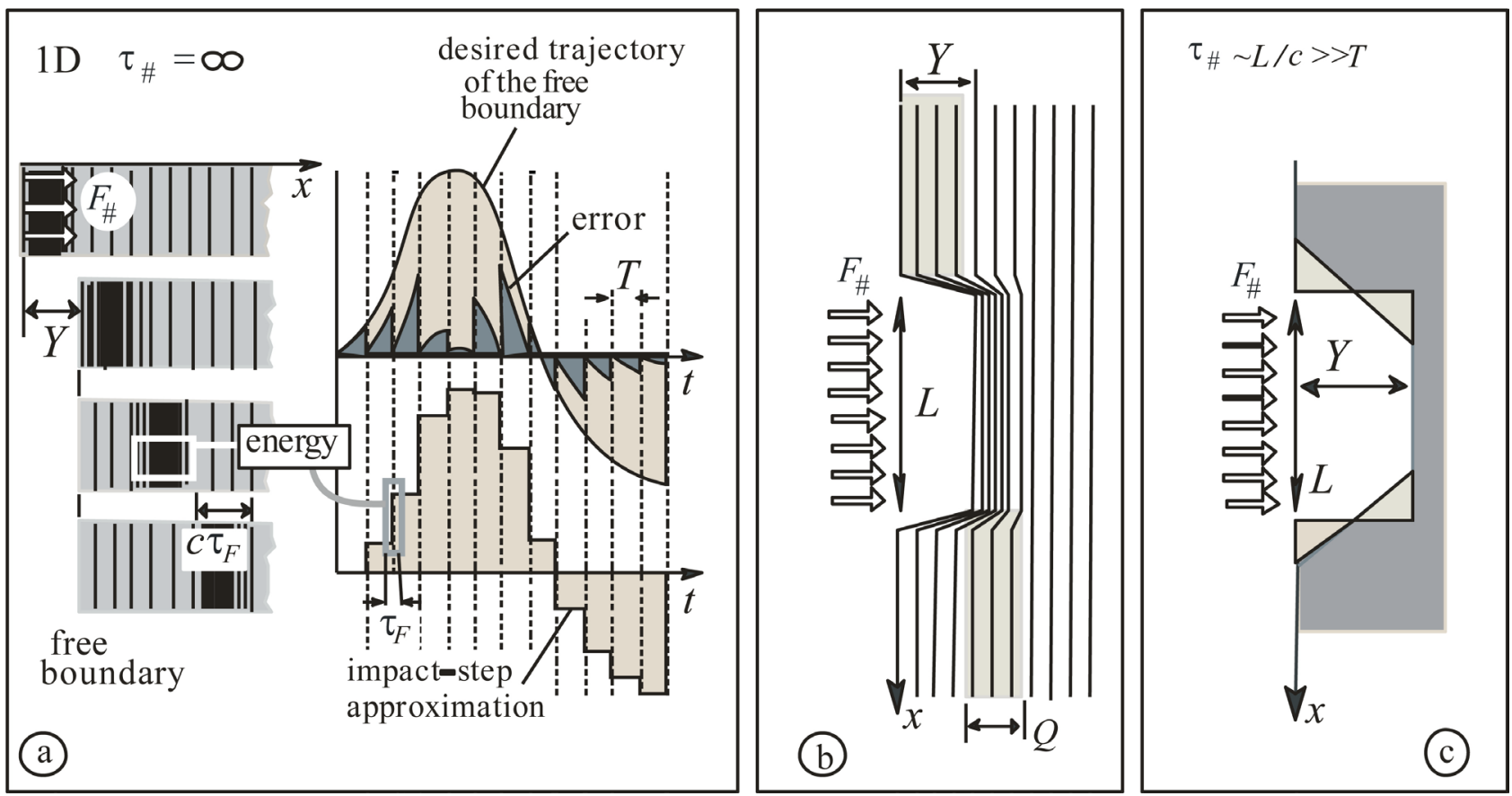

Figure 1. 1D impact (a). 2D-3D flat impact: structure of a flat impact imprint of one piston (b) and its spreading (space-time conversion) of the flat impact imprint of one piston (c).

shock force (pressure) $F_{\#}(t)$, that acts during the time interval $0<t \leq \tau_{F}$ $\left(F_{\#}=0\right.$ at $t<0$ and $t>\tau_{F}$ ). Such an ideal plasticity [3] of boundary $x=0$ is possible because the region (of thickness $Q=c \tau_{F}$ ) of elastic deformation runs to the right with sound speed $c$.

Linearity is guaranteed by the condition $|Y|<<|Q|$. Further, instead of the free end of the elastic rod, we consider the free plane boundary of a semi-infinite area $(-\infty<y, z<+\infty, x \geq 0)$ filled with a compressible medium with the same $\rho$ and $c(x<0$ is vacuum). We divide the plane $x=0$ into a set of regions in the form of infinite parallel strips: $|y-(L / 2) n|<L / 2,-\infty<z<+\infty$ or "pistons" numbered by $n=0, \pm 1, \pm 2, \pm 3, \ldots$. Suppose that we need to create a $\delta$-like distribution of normal displacements $U(y, t)$ that satisfies the condition $\int_{n L-(L / 2)}^{n L+(L / 2)} U(y, m T) d y / L=\bar{\varepsilon} \delta(n)$, where $m=0,1,2,3, \ldots, \delta=1$ for $n=0, \delta=0$ for $n \neq 0$. Thus at initial condition $U(y, 0)=0, U_{t}^{\prime}(y, 0)=0$ we need to apply the first impact of pressure $F_{\#}=\bar{\varepsilon} \rho c L / \tau_{F}$ to the strip $|y|<L / 2$. This pressure pulse (acting on the interval $0<t<\tau_{F}<L / c$ ) gives us the almost rectangular imprint of depth $\bar{\varepsilon}$ (deformation of boundary $x=0$ ). Due to the spreading of the imprint, its lifetime $\tau_{\#} \sim L / c$ is finite (Figure 1(b), Figure 1(c)). Therefore, the imprint must be supported by appropriate shock pumping (in time intervals $m T<t<m T+\tau_{F}, \quad m=1,2,3, \ldots$ ) of all the pistons with a time period $T$. There is the fact of fundamental importance that (due to the finite lifetime $\tau_{\#} \sim L / c$ of the print) pumping requires impacts which amplitude is the only a small part $\leq \gamma=c T / L$ of the first impact. The total background of pumping impacts is a small part $\leq \gamma /(1-\gamma)$ of the magnitude of the first strike. We call a 
combination $|Y|<<|Q|<<L$ a linear flat blow condition. Now (as in all linear problems), if we can form an almost constant time $\delta$-like distribution $U_{.}(y, t) \quad(\mathbf{r} \in S)$ of normal displacements, then we can also form an arbitrary given distribution with spatial resolution $\sim L$ and the scale $\sim 2 \pi / \omega_{\max } \gg T$ of temporal variability. Now we give a generalized definition of the piston as an element of an active shell on an arbitrary convex smooth closed surface $S$. Tangentially (Figure 3(a)) the active shell (spaced between the surfaces $S_{B}$ and $S$ ) is lumped into a set of plane pistons with contours of convex polygons. Each piston (of characteristic linear scale $\sim L$ ) corresponds to some area $\hat{\sigma}_{S}$ of the surface $S_{B}$ (or $S$ ), as well as the coordinate $\overline{\mathbf{R}}=\left(\sigma_{S}\right)^{-1} \int_{\mathbf{r} \in \hat{\sigma}_{S}} \mathbf{r} d \hat{\sigma}_{S}(\mathbf{r})$ of the center (where $\sigma_{S}=\int_{\mathbf{r} \in \hat{\sigma}_{S}} d \hat{\sigma}_{S}(\mathbf{r})$ is piston square) to which all control and measuring signals are addressed. Under the needed $\bar{U}_{\text {. }}\left(\overline{\mathbf{R}}, t_{n}\right)$ and actual (measured) $\bar{U}_{\otimes}\left(\overline{\mathbf{R}}, t_{n}\right)$ normal displacements of the piston (the center of which is at the point $\overline{\mathbf{R}}$ ) we mean the quantities averaged over the area $\hat{\sigma}_{S}$ of the piston. Above we assumed that for the impacts creation we have some unlimited source of mechanical impulse or support (vibrostat). Below we show that it is possible to synthesize a needed distribution $U_{\bullet}(\mathbf{r}, t)$ of the normal displacements of the surface $S$ without mechanical support too.

\subsection{Transparent Supportless Unidirectional Sources}

Let's consider the piezoelectric plane layer $-h<x \leq 0$ with the same (for simplicity) $\rho$ and $c$ as at $x \leq-h$ and at $x>0$. Pulse of voltage $\bar{\varphi}(t)$ with duration $\tau_{F}<<h / c$ creates two normal displacement pulses (of mutually opposite polarity and of the length $h$ ) running to the left and to the right (see Figure 2(a)). Piezoelectric forces $\pm F_{\#}$ and $\mp F_{\#}$ of compression (tension) are mutually balanced and need not mechanical support. Next we consider two piezoelectric layers $-2 h<x \leq-h$ and $-h<x \leq 0$ excited by voltage pulses $\bar{\varphi}(t)$ and $\overline{\bar{\varphi}}(t)$ (Figure 2(b)) of the same duration but separated in time from each other by the delay $h / c$ and with mutually opposite polarity. It is easy to see (Figure 2(c)) that at $x \leq-2 h$ pulses of normal displacement created by voltage pulses (applied to above layers) are mutually compensated. But at $x>0$ these pulses of normal displacement form the bipolar wavelet with duration $3 \mathrm{~h} / \mathrm{c}$ and pause of duration $h / c$ between pulses. This wavelet is created also without any mechanical support [3].

If the impact duration $\left(\tau_{F}<<T\right)$ is negligible, then we can write the wavelet $\Psi(\xi) \quad(\xi=x-c t$, running to right) of single-direction radiation in the following form: $\Psi(\xi)=\{I[\xi]-I[\xi-(1 / 2) T]\}-\{I[\xi-T]-I[\xi-(3 / 2) T]\}$, where $T=2 h / c, I(\xi)=1$ at $\xi>0, I(\xi)=0$ at $\xi \leq 0$. Summarizing these wavelets with amplitudes $Y_{n}$ and shifted with respect to each other by time distance $T=2 h / c$, we can form a sequence of hooked wave with duration $3 h / c$ of each: $Y_{0} \Psi(t)+Y_{1} \Psi(t-T)+Y_{2} \Psi(t-2 T)+\cdots$ (Figure 2(d)). In this case we obtain a sequence of pulses with periodic pauses of duration $h / c$. Therefore, to create a needed normal displacement $\bar{U}_{\mathbf{.}}(\overline{\mathbf{R}}, t)$ of the piston at the $n$-th step, 


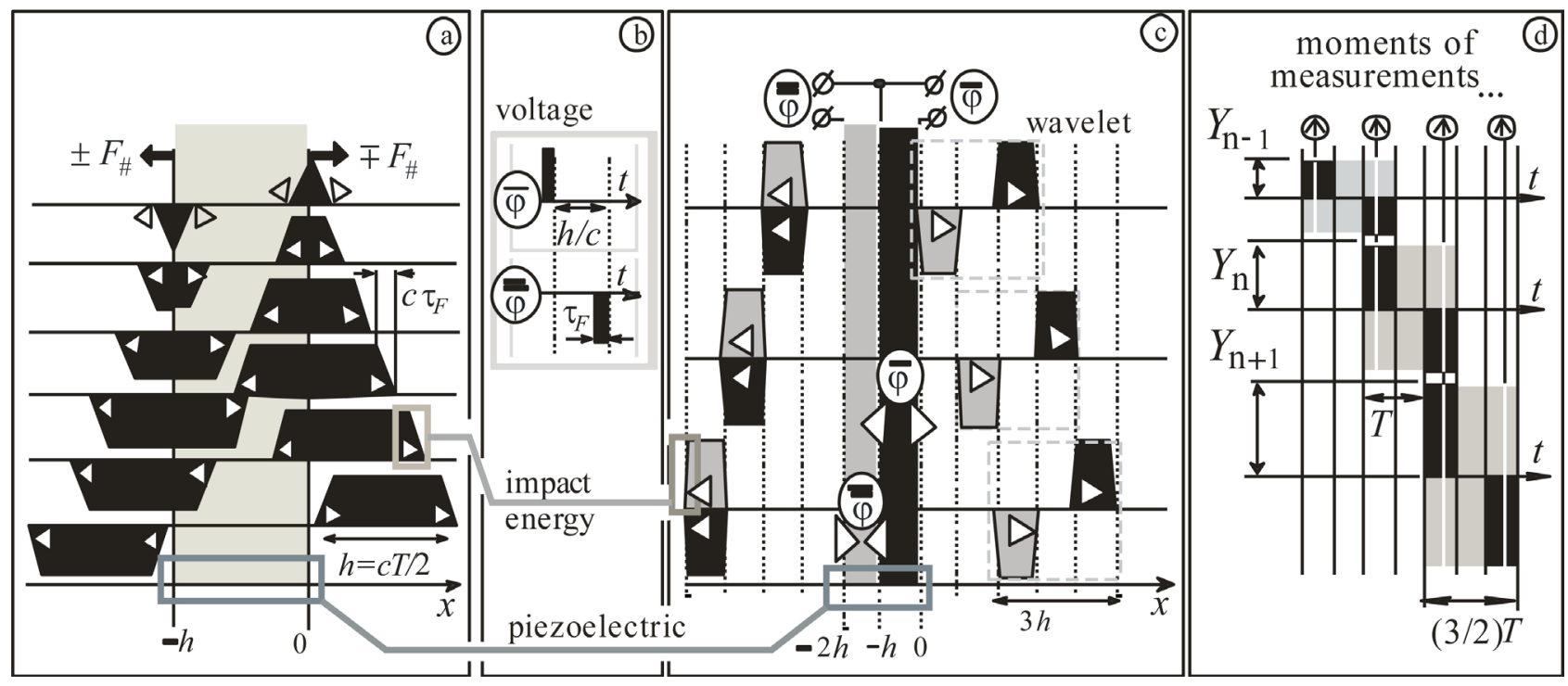

Figure 2. Supportless flat impact. Formation of a normal displacement pulse (instant spatial distributions at different moments of time). Single-layer piezoelectric (a). Voltage excitation pulses $\bar{\varphi}(t), \overline{\bar{\varphi}}(t)$ of a two-layer piezoelectric (b). Two-layer piezoelectric dynamics (c). Summarizing the wavelets in time at boundary $x=0 \quad$ (d).

the amplitude of the wavelet must be double, in order to provide, on average, the desired value of the normal displacement on the period $T=2 \mathrm{~h} / \mathrm{c}$. Thus, we obtain the following expression (control algorithm) of the current amplitude $Y_{n}$ of the wavelet through via amplitude $Y_{n-1}$ of the previous and measured displacement $\bar{U}_{\otimes}\left(\overline{\mathbf{R}}, t_{n-1}\right)$ of the piston, as well as the required displacement $\bar{U}_{\bullet}\left(\overline{\mathbf{R}}, t_{n}\right)$ value

$$
Y_{n}=Y_{n-1}+2\left[\bar{U} \cdot\left(\overline{\mathbf{R}}, t_{n}\right)-\bar{U}_{\otimes}\left(\overline{\mathbf{R}}, t_{n-1} ; Y_{0}, Y_{1}, Y_{2}, \ldots, Y_{n-1}\right)\right],
$$

where $t_{n}=n T, n=0,1,2, \ldots$ The above-mentioned spreading of the imprints of the blows (for compensation of which is necessary the impact pumping) is contained in the measured quantity $\bar{U}_{\otimes}\left(\overline{\mathbf{R}}, t_{n-1} ; Y_{0}, Y_{1}, Y_{2}, \ldots, Y_{n-1}\right)$. Note that an attempt to synthesize a desired value $\bar{U}_{\mathbf{v}}(\overline{\mathbf{R}}, t)$ using bipolar wavelets $\Psi(t)$ means that the amplitude $Y_{n}$ of the wavelets is proportional to the integral of the quantity $\bar{U}_{\mathbf{.}}\left(\overline{\mathbf{R}}, t_{n}\right)-\bar{U}_{\otimes}\left(\overline{\mathbf{R}}, t_{n-1}\right)$. Thus, neither the measured displacement $\bar{U}_{\otimes}(\overline{\mathbf{R}}, t)$ nor the desired value $\bar{U}_{\mathbf{0}}(\overline{\mathbf{R}}, t)$ should contain time-constant components. To maintain stability, it is necessary to exclude the constant component of the signals $\bar{U}_{\otimes}(\overline{\mathbf{R}}, t)$ and $\bar{U}_{\mathbf{.}}(\overline{\mathbf{R}}, t)$, i.e. pass them through a non-distorting differential filter with a time scale $\tau_{d}>>\lambda_{\max } / c$. Now we must note that slow desired trajectory $\bar{U}_{\cdot}(\overline{\mathbf{R}}, t)$ of piston in time (with time scale $\tau_{\max }=\lambda_{\max } / 2 c$ ) requires significant value of wavelet magnitude at some maximum amplitude $A_{\text {max }}$ of particle displacement in the waves to be damped. So the condition of linear flat impact becomes the following: $\left(\tau_{\max } / T\right) A_{\max }<<c \tau_{F}<<c T<<L$.

\subsection{Measuring}

Figure 3 presents the structure of active shall. Tangential structure of active shall is presented in Figure 3(a). Transversally (Figure 3(b)) each piston is presented by two piezoelectric layers. It is necessary very quickly and accurately estimate 


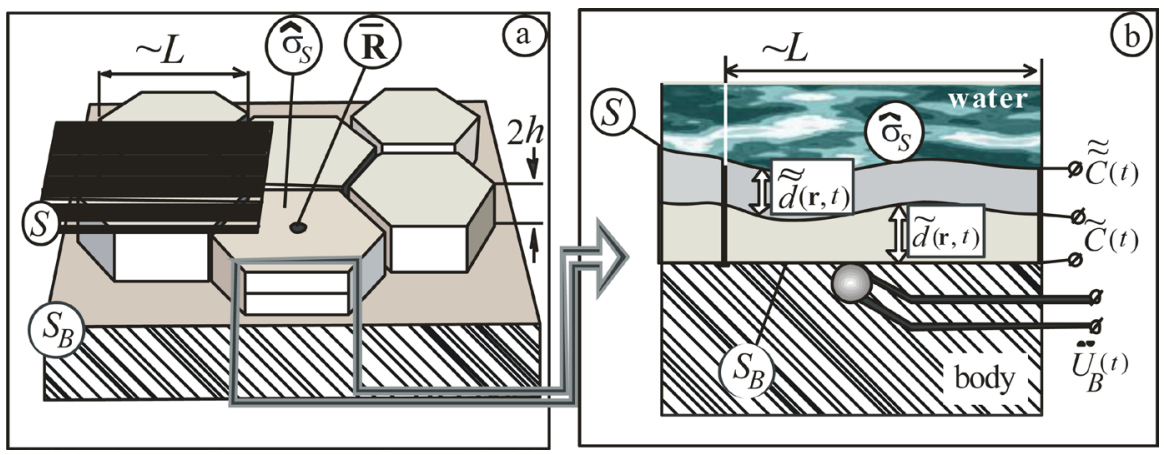

Figure 3. Tangential (a) and transverse (b) structure of active shell.

(between piezoelectric impacts) the current average piston (with the center in the point $\overline{\mathbf{R}} \in S$ ) area $\mathbf{r} \in \hat{\sigma}_{S} \subset S$ particle displacement $\bar{U}_{\otimes}(\overline{\mathbf{R}}, t)=U_{B}(\overline{\mathbf{R}}, t)+\tilde{U}(\overline{\mathbf{R}}, t)+\tilde{\tilde{U}}(\overline{\mathbf{R}}, t)$, where $U_{B}(\overline{\mathbf{R}}, t)$-slow displacement of surface $S_{B}$, where $\{\tilde{U}(\overline{\mathbf{R}}, t), \tilde{\tilde{U}}(\overline{\mathbf{R}}, t)\}=\left(\sigma_{S}\right)^{-1} \int\{\tilde{d}(\mathbf{r}, t), \tilde{\tilde{d}}(\mathbf{r}, t)\} d \hat{\sigma}(\mathbf{r})$ are instant spatial average in the area $\mathbf{r} \in \hat{\sigma}_{S}$ of the $e^{\hat{\sigma}_{\text {thicknesses }}} \tilde{d}(\mathbf{r}, t), \tilde{\tilde{d}}(\mathbf{r}, t)$ of the metallized layers of the piezoelectric. The smoothness of the distribution of displacements of the surface $S_{B}$ in space is guaranteed by the condition $\ell_{\min }>L$, where $\ell_{\max }$ is maximum spatial scale of displacement distribution on the surface $\left.S_{B}\right)$. Therefore, $U_{B}(\overline{\mathbf{R}}, t)$ can be used without spatial averaging over the piston pad. Then we need to measure the instant capacitances $\tilde{C}(t)$ and $\tilde{\tilde{C}}(t)$ of the flat capacitors (dielectric layers) with varying thicknesses $\{\tilde{d}(\mathbf{r}, t), \tilde{\tilde{d}}(\mathbf{r}, t)\}=h+\left\{\tilde{d}_{\sim}(\mathbf{r}, t), \tilde{\tilde{d}}_{\tilde{\tilde{d}}}(\mathbf{r}, t)\right\} \quad$ (where the variable components are relatively small, i.e. $\left|\tilde{d}_{\sim}\right|<<,\left|\tilde{\tilde{d}}_{\sim}\right|<<$ ) of the dielectric (piezoelectric) layers: $\{\tilde{C}(t), \tilde{\tilde{C}}(t)\}=\left(\varepsilon_{0} \varepsilon\right)^{-1} \int d \hat{\sigma}_{S}(\mathbf{r}) /\{\tilde{d}(\mathbf{r}, t), \tilde{\tilde{d}}(\mathbf{r}, t)\}$, where $\varepsilon_{0}$-dielectric constant of vacuum, $\varepsilon$-relafî́, permittivity of piezoelectric. Now we write down the needed quantities $\{\tilde{U}(\overline{\mathbf{R}}, t), \tilde{\tilde{U}}(\mathbf{R}, t)\}=-h\left[\{\tilde{C}(t), \tilde{\tilde{C}}(t)\}-C_{0}\right] / C_{0}$, where $C_{0}=\sigma_{S} /\left(\varepsilon_{0} \varepsilon h\right)$. These spatial averaging electric operations can be performed almost instantaneously. Inertial accelerometer with output signal $\ddot{U}_{B}(t)$ (i.e. the 2-nd derivative of normal displacement $U_{B}(t)$ of body surface) is placed immediately under the center $\overline{\mathbf{R}}$ of piston. In the end, we write down the remaining component $U_{B}(\overline{\mathbf{R}}, t)=\int d \xi \hat{\int} \hat{\bar{L}} \ddot{U} L_{B}(\eta) d \eta$, where $\hat{\bar{L}}$ means 3-fold processing by differentiating chain ${ }^{0}$ with time scale $\tau_{d}>>\lambda_{\max } / c$.

\section{Scattering Suppression}

Suppose that body's radiation is already suppressed by the system described in Section 2. Further suppose that in area of compressible medium (with mass density $\rho$ and sound speed $c$, identical with outer medium) delineated by surface $S$ we know the particle displacement field $\mathbf{U}_{I}(\mathbf{r}, t)$ created by the incident waves. Scattering field does not arise if we create on the outer surface of active shell the distribution $U_{\bullet}(\mathbf{r}, t)=\mathbf{n}(\mathbf{r}) \mathbf{U}_{I}(\mathbf{r}, t)$ of normal displacements $U_{.}(\mathbf{r}, t) \quad(\mathbf{n}(\mathbf{r})$ is the outer normal to the surface $S$ in its point $\mathbf{r} \in S,|\mathbf{n}(\mathbf{r})|=1)$ which coincides with normal component of particle displacement in the incident waves field. 


\subsection{Incident Waves}

Further we assume that incident wave field $P_{I}(\mathbf{r}, t)=\sum_{n=1}^{N_{I}} P_{I n}(\mathbf{r}, t)$ of pressure represents the finite set of $N_{I} \geq 1$ planar waves $P_{I n}(\mathbf{r}, t)=\Theta_{I n}\left[t-\left(\mathbf{w}_{n}, \mathbf{r}\right) c^{-1}\right]$, with vectors $\mathbf{w}_{n} \quad\left(\left|\mathbf{w}_{n}\right|=1\right)$ of propagation direction and profiles $\Theta_{I n}(\xi)$ with leading edges. this means that there is a point $\xi_{n}$, for which the following condition is satisfied: $\Theta_{I n}(\xi)=0$ at any $\xi<\xi_{n}, \Theta_{I n}(\xi) \neq 0$ at $\xi_{n}<\xi<\xi_{n}+\left(\lambda_{\min } / 4\right)$, where $\lambda_{\min }$ is the minimum length of the wave to be damped.

\subsection{Spacing of Microphones}

All the microphones are placed in points $\mathbf{r}=\mathbf{r}$. $\quad(\mathbf{r}$. means the coordinate of any microphone). In addition, all microphones are placed by pairs in points $\mathbf{r}_{\mathbf{0}}=\mathbf{R}$ (farer to $S$ and called "title microphone") and $\mathbf{r}_{\mathbf{0}}=\mathbf{R}_{+}$(nearer to $S$ and called "reference microphone") on the normal $\mathbf{n}=\mathbf{n}(\mathbf{R})$ to a smooth convex surface $S$ with distance $D$ between them (see $\mathbf{n}_{A 1}, \mathbf{n}_{B 1}, \quad \mathbf{n}_{C 1}$ in Figure 4(a)). So vector $\mathbf{R}_{+}-\mathbf{R}$ is parallel to normal $\mathbf{n}=\mathbf{n}(\mathbf{R})$ and $\mathbf{R}_{+}=\mathbf{R}-D \mathbf{n}(\mathbf{R})$. Distance between reference microphone (in the point $\mathbf{r}_{\mathbf{0}}=\mathbf{R}_{+}$) and surface $S$ we will denote $H=H\left(\mathbf{R}_{+}\right)>>h, D$. Points $\mathbf{R}$ presents the vertices of some convex polyhedron. Further, we will not designate microphones with specific numbers to simplify the presentation. At some initial moment, the leading edges of all the incident waves have not yet reached any title microphone. Note that all microphones should be insensitive to pressure fluctuations at a very high frequencies $\geq 2 \pi / T$ corresponding to the surface $S$ flat impacts (Section 2).

\subsection{Arrival of 1 -st Incident Wave}

Radiation of internal sources within surface $S_{B}$ is assumed suppressed by the

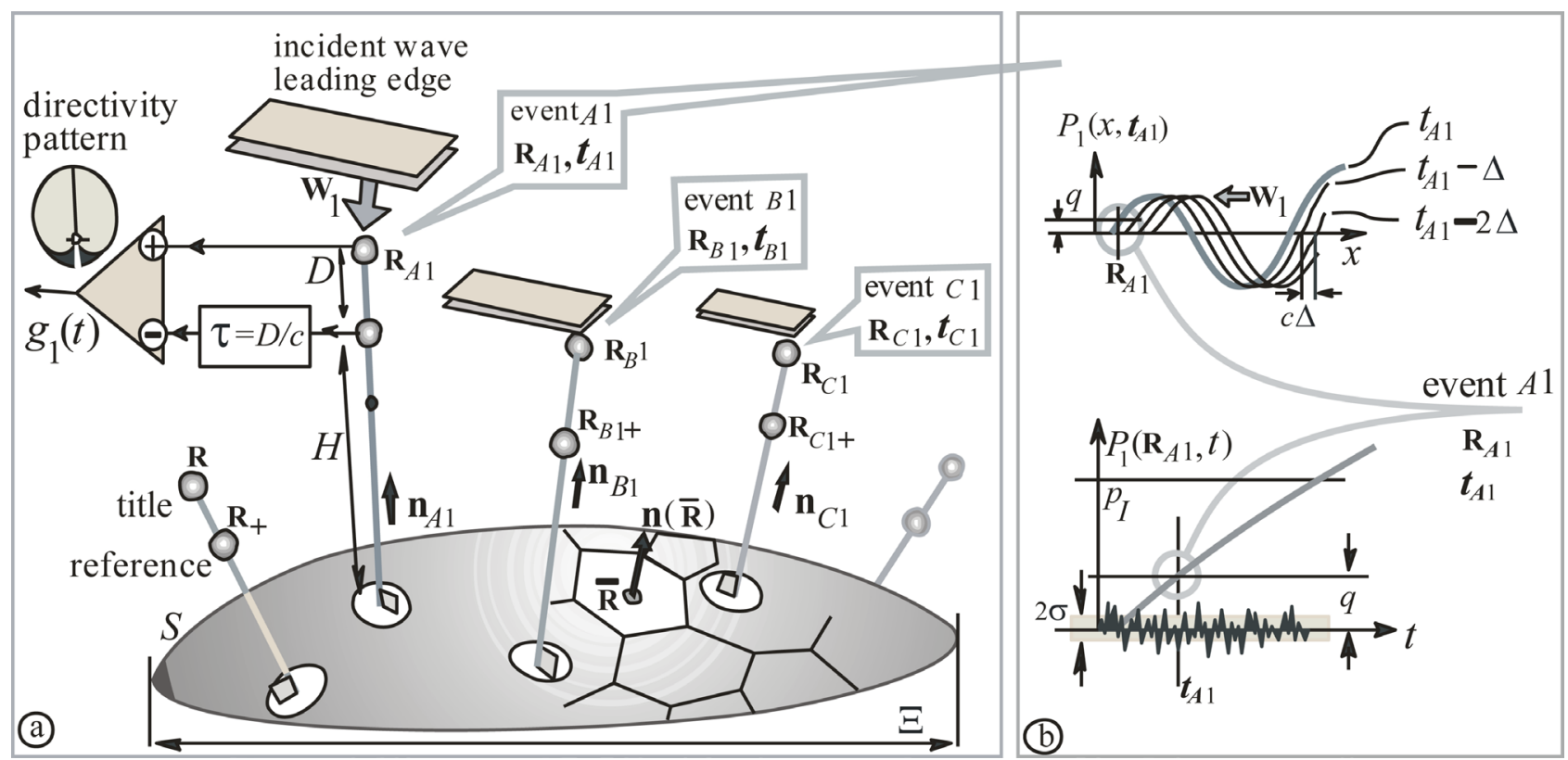

Figure 4. The geometry of scattering suppression problem (a), space-time event of threshold level crossing by leading edge of incident wave (b). 
means described in Section 2. All microphones are waiting for the first incident wave arrival from unknown direction $\mathbf{w}_{1}$ (output pressure signals of all microphones are denoted by $\left.P_{1}\left[\mathbf{r}_{\bullet}, t\right]\right)$. Leading edge of some plane incident wave (we will call this wave the 1 -st incident wave with direction vector) achieves some microphone spaced the in the point $\mathbf{r}_{\mathbf{v}}=\mathbf{R}_{A 1}$ at some moment $t=t_{A 1}$. This is some space-time node (or event $A 1$ ): module $\left|P_{1}\left(\mathbf{R}_{A 1}, t\right)\right|$ of output signal of microphone spaced in the point $\mathbf{r}_{\mathbf{0}}=\mathbf{R}_{A 1}$ sound pressure $P_{1}\left[\mathbf{R}_{A 1}, t\right]$ crosses at the first time some level $q$ from $\left|P_{1}\left[\mathbf{R}_{A 1}, t\right]\right|<q$ to $\left|P_{1}\left[\mathbf{R}_{A 1}, t\right]\right|>q$ at the moment $t=t_{A 1}$. We notice a similar event $B 1$ later on some microphone with coordinate $\mathbf{r}_{\bullet}=\mathbf{R}_{B 1}$ at some moment $t=t_{B 1} \geq t_{A 1}$ : module of pressure $P_{1}\left[\mathbf{R}_{B 1}, t\right]$ crosses at the first time some level $q$ from $\left|P_{1}\left[\mathbf{R}_{B 1}, t\right]\right|<q$ to $\left|P_{1}\left[\mathbf{R}_{B 1}, t\right]\right|>q$ at the moment $t=t_{B 1}$. And the next similar event $C 1$ in the point $\mathbf{r}_{\mathbf{v}}=\mathbf{R}_{C 1}$ at some moment $t=t_{C 1} \geq t_{B 1}$ : module of pressure $P_{1}\left[\mathbf{R}_{C 1}, t\right]$ crosses at the first time some level $q$ from $\left|P_{1}\left[\mathbf{R}_{C 1}, t\right]\right|<q$ to $\left|P_{1}\left[\mathbf{R}_{C 1}, t\right]\right|>q$ at the moment $t=t_{C 1}$. Assuming below the ratio $p_{I} \gg>q>\sigma$ (where $p_{I}$ is the characteristic amplitude of the pressure in the incident wave, and $\sigma$-the mean square deviation of background noise signal, see Figure 4(b)), we obtain the propagation vector $\mathbf{w}_{1}=\mathbf{w}_{1}(A 1, B 1 ; C 1)$ of the 1-st incident wave from system of equations $\left|\left(\mathbf{R}_{B 1}-\mathbf{R}_{A 1}\right) \mathbf{w}_{1}\right|=c\left(t_{B 1}-t_{A 1}\right)$ and

$\left|\left(\mathbf{R}_{B 1}-\mathbf{R}_{A 1}\right) \mathbf{w}_{1}\right|=c\left(t_{B 1}-t_{A 1}\right)$. Usually incident wave is sufficiantly powerful because one need to detect scattered wave at a large distance from the body. Now for stability of the active system we need to form signal of the incident wave pressure, insensitive (simplest spatial filtration) to waves scattered by the surface $S$ due to possible violation of the condition $u_{S}(\mathbf{r}, t)=\mathbf{n}_{S}(\mathbf{r}) \mathbf{U}_{I}(\mathbf{r}, t)$. To do this, we will form da leading pair of microphones or a combination $g_{1}(t)=\hat{L} P_{1}\left[\mathbf{R}_{A 1}, t\right]-\hat{L} P_{1}\left[\mathbf{R}_{A 1+}, t-\tau\right]$ of signals $P_{1}\left[\mathbf{R}_{A 1}, t\right]$ and $P_{1}\left[\mathbf{R}_{A 1+}, t-\tau\right]$ two microphones (in points $\mathbf{R}_{A 1}$ (title microphone) and $\mathbf{R}_{A 1+}=\mathbf{R}_{A 1}-D \mathbf{n}\left(\mathbf{R}_{A 1}\right)$ (reference microphone) with cardioid directivity pattern having zero in the direction to the surface $S$ (see Figure 4(a)). Here $\tau=D / c$ -delay, $\hat{L}$-linear filter, undistorting signals at frequencies $\omega_{\min } \leq \omega \leq \omega_{\max }$ $\left(\hat{L} P_{I}=P_{I}\right)$ and opaque for frequencies $0<\omega<<\omega_{\min }, \omega>2 \pi / T>>\omega_{\max }$. Under the condition $D<<2 \pi c / \omega_{\max }$ we obtain $g_{1}(t)=\tau(\partial / \partial t)\left\{\hat{L} P_{1}\left[R_{A 1}, t\right]\right\}$, and for $P_{1}\left[\mathbf{R}_{A 1}, t\right]=P_{I 1}\left[\mathbf{R}_{A 1}, t\right] \quad\left(1{ }^{\text {st }}\right.$ incident wave) we obtain $P_{I 1}\left[\mathbf{R}_{A 1}, t\right]=P_{1}\left[\mathbf{R}_{C 1}, t_{A 1}\right]+\tau^{-1}\left[1-\left(\mathbf{n}_{A 1} \mathbf{w}_{1}\right)\right]^{-1} \int_{t_{C 1}}^{t} g_{1}(\xi) d \xi \quad$ (note that we have $\left(\mathbf{n}_{A 1} \mathbf{w}_{1}\right)<0$ for any incident plane wave). Knowing the pressure field $P_{I 1}$ of the first incident plane wave at a point $\mathbf{R}_{A 1}$ at time $t>t_{A 1}$, we can determine the pressure field at any point $\mathbf{r}$ (satisfying the condition $\left(\mathbf{r}-\mathbf{R}_{A 1}\right) \mathbf{w}_{1}>0$ ) at time $t>t_{A 1}$. In addition, we can determine the normal displacement $u_{S}(\mathbf{r}, t)$ of the surface $S$ under which the condition $u_{S}(\mathbf{r}, t)=\mathbf{n}_{S}(\mathbf{r}) \mathbf{U}_{I 1}(\mathbf{r}, t)$ will be satisfied (the displacement field $\mathbf{U}_{I 1}(\mathbf{r}, t)$ in the first incident plane wave in infinite homogeneous compressible medium) and scattering will not arise. More precisely, we will establish a normal average over the area $\hat{\sigma}_{S}$ of the piston (with the center at the point $\overline{\mathbf{R}}$ of the surface $S$ ) displacement 


$$
\bar{U}_{\bullet}(\overline{\mathbf{R}}, t+T)=\bar{U}_{\bullet}(\overline{\mathbf{R}}, t)+\delta_{1} P_{I 1}\left[\mathbf{R}_{A 1}, t-\alpha_{1}\right]
$$

(correcting it for a period of duration $T$, i.e. pressure-velocity-displacement), where $\delta_{1}=(T / \rho c)\left(\mathbf{w}_{1} \mathbf{n}(\overline{\mathbf{R}})\right), \quad \alpha_{1}=\mathbf{w}_{1}\left(\overline{\mathbf{R}}-\mathbf{R}_{A 1}\right) / c$. After inserting (2) into (1), where $t+T=t_{n}$, the scattering does not occur, then the field of the first incident wave $P_{I 1}[\mathbf{r}, t]$ passes without distortion through the region of space occupied by the body and bounded by the surface $S$. And this means that the pressure field of the first incident wave $P_{I 2}\left[\mathbf{r}_{\text {。 }}, t\right]$ (for $t>t_{C 1}$ ) can be subtracted from the signals of all microphones except for the leading pair (at points $\mathbf{R}_{A 1}$ and $\mathbf{R}_{A 1+}$ ) of the first incident wave. The sound pressure on the microphones at the points $\mathbf{r}_{.} \neq \mathbf{R}_{A 1}, \mathbf{R}_{A 1+}$ will now be denoted as $P_{2}\left[\mathbf{r}_{\bullet}, t\right]=P_{1}\left[\mathbf{r}_{\bullet}, t\right]-P_{I 1}\left[\mathbf{R}_{A 1}, t-\beta_{1}\right]$, where $\beta_{1}=\mathbf{w}_{1}\left(\mathbf{r}_{\bullet}-\mathbf{R}_{A 1}\right) / c$. Thus, we prepared the system for capturing a second plane incident wave, with respect to which we will assume that for the first points of contact of the leading edge with the microphones there will be points $\mathbf{r} .=\mathbf{R} \neq \mathbf{R}_{A 1}, \mathbf{R}_{A 1+}$ of placement of the title microphones. The microphones at the points $\mathbf{r}_{\bullet} \neq \mathbf{R}_{A 1}, \mathbf{R}_{A 1+}$ became deaf (insensitive) to the first incident wave. Therefore, one can apply the logical procedure described above to the signals $P_{2}\left[\mathbf{r}_{.}, t\right]$ (three events $A 2\left(t_{A 2}, \mathbf{R}_{A 2}\right), B 2\left(t_{B 2}, \mathbf{R}_{B 2}\right), C 2\left(t_{C 2}, \mathbf{R}_{C A 2}\right)$ ). And so on. Below we give briefly a sequence of next functional steps.

\subsection{Arrival of 2-nd Incident Wave}

Event $A 2: t=t_{A 2} ; \mathbf{r}_{0}=\mathbf{R}_{A 2} ;$ crossing $\left|P_{2}\right|<q \Rightarrow\left|P_{2}\right|>q$. Event $B 2: t=t_{B 2}$; $\mathbf{r}_{\mathbf{0}}=\mathbf{R}_{B 2} ;$ crossing $\left|P_{2}\right|<q \Rightarrow\left|P_{2}\right|>q$. Event $C 2: t=t_{C 2} ; \mathbf{r}_{\mathbf{0}}=\mathbf{R}_{C 2} ;$ crossing $\left|P_{2}\right|<q \Rightarrow\left|P_{2}\right|>q$.

$\left|\left(\mathbf{R}_{B 2}-\mathbf{R}_{A 2}\right) \mathbf{w}_{2}\right|=c\left(t_{B 2}-t_{A 2}\right),\left|\left(\mathbf{R}_{B 2}-\mathbf{R}_{A 2}\right) \mathbf{w}_{2}\right|=c\left(t_{B 2}-t_{A 2}\right)$,

$\mathbf{w}_{2}=\mathbf{w}_{2}(A 2, B 2, C 2), g_{2}(t)=\hat{L} P_{2}\left[\mathbf{R}_{A 2}, t\right]-\hat{L} P_{2}\left[\mathbf{R}_{A 2+}, t-\tau\right]$,

$\delta_{2}=(T / \rho c)\left(\mathbf{w}_{2} \mathbf{n}(\overline{\mathbf{R}})\right), \quad \alpha_{2}=\mathbf{w}_{2}\left(\overline{\mathbf{R}}-\mathbf{R}_{A 2}\right) / c$,

$P_{I 2}\left[\mathbf{R}_{A 2}, t\right]=P_{1}\left[\mathbf{R}_{A 2}, t_{C 2}\right]+\tau^{-1}\left[1-\left(\mathbf{n}_{A 2} \mathbf{w}_{2}\right)\right]^{-1} \int_{t_{C 2}}^{t} g_{2}(\xi) d \xi, \quad \beta_{2}=\mathbf{w}_{2}\left(\mathbf{r}_{\bullet}-\mathbf{R}_{A 2}\right) / c$,

$\bar{U}_{\bullet}(\overline{\mathbf{R}}, t+T)=\bar{U}_{\cdot}(\overline{\mathbf{R}}, t)+\delta_{1} P_{I 1}\left[\mathbf{R}_{A 1}, t-\alpha_{1}\right]+\delta_{2} P_{I 2}\left[\mathbf{R}_{A 2}, t-\alpha_{2}\right]$,

$P_{3}\left[\mathbf{r}_{\bullet}, t\right]=P_{3}\left[\mathbf{r}_{\bullet}, t\right]-P_{I 1}\left[\mathbf{R}_{A 1}, t-\beta_{1}\right]-P_{I 2}\left[\mathbf{R}_{A 2}, t-\beta_{2}\right]$, for $\mathrm{n}$

$\mathbf{r}_{\text {. } \neq} \neq \mathbf{R}_{A 1}, \mathbf{R}_{A 1+}, \mathbf{R}_{A 2}, \mathbf{R}_{A 2+}$.

\subsection{Arrival of 3-rd Incident Wave}

Event $A 3: t=t_{A 3} ; \quad \mathbf{r}_{0}=\mathbf{R}_{A 3} ;$ crossing $\left|P_{3}\right|<q \Rightarrow\left|P_{3}\right|>q$. Event $B 3: t=t_{B 3}$; $\mathbf{r} .=\mathbf{R}_{B 3} ;$ crossing $\left|P_{3}\right|<q \Rightarrow\left|P_{3}\right|>q$. Event $C 3: t=t_{C 3} ; \quad \mathbf{r}_{\mathbf{.}}=\mathbf{R}_{C 3} ;$ crossing $\left|P_{3}\right|<q \Rightarrow\left|P_{3}\right|>q$.

$$
\begin{aligned}
& \left|\left(\mathbf{R}_{B 3}-\mathbf{R}_{A 3}\right) \mathbf{w}_{3}\right|=c\left(t_{B 3}-t_{A 3}\right), \\
& \left|\left(\mathbf{R}_{B 3}-\mathbf{R}_{A 3}\right) \mathbf{w}_{3}\right|=c\left(t_{B 3}-t_{A 3}\right) \Rightarrow \mathbf{w}_{3}=\mathbf{w}_{3}(A 3, B 3, C 3), \\
& g_{3}(t)=\hat{L} P_{3}\left[\mathbf{R}_{A 3}, t\right]-\hat{L} P_{3}\left[\mathbf{R}_{A 3+}, t-\tau\right], \quad \delta_{3}=(T / \rho c)\left(\mathbf{w}_{3} \mathbf{n}(\overline{\mathbf{R}})\right), \\
& \alpha_{3}=\mathbf{w}_{3}\left(\overline{\mathbf{R}}-\mathbf{R}_{A 3}\right) / c, P_{I 3}\left[\mathbf{R}_{A 3}, t\right]=P_{3}\left[\mathbf{R}_{A 3}, t_{C 3}\right]+\tau^{-1}\left[1-\left(\mathbf{n}_{A 3} \mathbf{w}_{3}\right)\right]^{-1} \int_{t_{C 3}}^{t} g_{3}(\xi) d \xi, \\
& \beta_{3}=\mathbf{w}_{3}\left(\mathbf{r}_{\bullet}-\mathbf{R}_{A 3}\right) / c, \\
& \bar{U}(\overline{\mathbf{R}}, t+T)=\bar{U} \cdot(\overline{\mathbf{R}}, t)+\delta_{1} P_{I 1}\left[\mathbf{R}_{A 1}, t-\alpha_{1}\right]+\delta_{2} P_{I 2}\left[\mathbf{R}_{A 2}, t-\alpha_{2}\right]+\delta_{3} P_{I 3}\left[\mathbf{R}_{A 3}, t-\alpha_{3}\right],
\end{aligned}
$$


$P_{4}\left[\mathbf{r}_{\bullet}, t\right]=P_{3}\left[\mathbf{r}_{\bullet}, t\right]-P_{I 1}\left[\mathbf{R}_{A 1}, t-\beta_{1}\right]-P_{I 2}\left[\mathbf{R}_{A 2}, t-\beta_{2}\right]-P_{I 3}\left[\mathbf{R}_{A 3}, t-\beta_{3}\right]$, for $\mathbf{r}_{.} \neq \mathbf{R}_{A 1}, \mathbf{R}_{A 1+}, \mathbf{R}_{A 2}, \mathbf{R}_{A 2+}, \mathbf{R}_{A 3}, \mathbf{R}_{A 3+}$.

\section{Conclusion}

The main results of this work are the following: 1) transparent supportless unidirectional sources of acoustical wavelets (Section 2), 2) causal sequence of operations to reconcile the normal displacements of the protected surface with the incident waves (Section 3). The presented results are a consequence of the problem formulation with initial conditions and could not be obtained in the widespread stationary monochromatic mathematical model of the problem with complex amplitudes (magnitude $A$, frequency $\omega$, phase $\varphi$, i.e. $A \exp [i(\omega t+\varphi)]$ ) of the fields (like in [2] for instance). The monochromatic representation leaves out of view some important situations, such as spatio-temporal labyrinths, where the control algorithm (like "Maxwell's demon") operates extremely quickly on the spatial microscales, and this leads to macroscopic results for long slow waves [3].

\section{Conflicts of Interest}

The authors declare no conflicts of interest regarding the publication of this paper.

\section{References}

[1] Bi, Y.F., Jia, H., Sun, Z.Y., Yang, Y.Z., Zhao, H. and Yang, J. (2018) Experimental Demonstration of Three-Dimensional Broadband Underwater Acoustic Carpet Cloak. Appl. Phys. Lett., 112. https://doi.org/10.1063/1.5026199

[2] Cummer, S.A., Popa, B.I., Schurig, D., Smith, D.R., Pendry, J., Rahm, M. and Starr, A. (2008) Scattering Theory Derivation 3D Acoustic Cloacking Shell. Physical Review Letters, 100.

[3] Arabadzhi, V.V. (2011) Solutions to Problems of Controlling Long Waves with the Help of Micro-Structure Tools. Bentham Science Publishers. 